University of Nebraska - Lincoln

DigitalCommons@University of Nebraska - Lincoln

Faculty Publications from Nebraska Center for

Materials and Nanoscience, Nebraska Center Materials and Nanoscience

for (NCMN)

December 2004

\title{
Attenuation of ultrasonic waves in rolled metals
}

Liyong Yang

University of Nebraska - Lincoln, lyang4@unl.edu

Joseph A. Turner

University of Nebraska - Lincoln, jaturner@unl.edu

Follow this and additional works at: https://digitalcommons.unl.edu/cmrafacpub

Part of the Nanoscience and Nanotechnology Commons

Yang, Liyong and Turner, Joseph A., "Attenuation of ultrasonic waves in rolled metals" (2004). Faculty Publications from Nebraska Center for Materials and Nanoscience. 68.

https://digitalcommons.unl.edu/cmrafacpub/68

This Article is brought to you for free and open access by the Materials and Nanoscience, Nebraska Center for (NCMN) at DigitalCommons@University of Nebraska - Lincoln. It has been accepted for inclusion in Faculty Publications from Nebraska Center for Materials and Nanoscience by an authorized administrator of DigitalCommons@University of Nebraska - Lincoln. 


\title{
Attenuation of ultrasonic waves in rolled metals
}

\author{
Liyong Yang and Joseph A. Turner ${ }^{\mathrm{a})}$ \\ Department of Engineering Mechanics, Center for Materials Research and Analysis, W317.4 Nebraska \\ Hall, University of Nebraska-Lincoln, \\ Lincoln, Nebraska 68588-0526
}

(Received 12 March 2004; revised 31 August 2004; accepted 1 September 2004)

\begin{abstract}
Scattering of ultrasonic waves in polycrystals with texture is studied in this article. The attenuations of the three wave modes are determined as a function of dimensionless frequency and propagation direction, respectively, for given orientation distribution coefficients (ODCs). The calculation is done in the case of a statistically orthorhombic sample made up of cubic crystallites. The wave propagation and scattering model is formulated by the Dyson equation using an anisotropic Green's function approach. Within the limits of the first-order smoothing approximation, the Dyson equation is solved in the spatial Fourier transform domain. The results presented are shown to be directional dependent, frequency dependent, and especially dependent on the texture coefficients (ODCs) for the quasilongitudinal and two quasishear waves. The theoretical results presented may be used to improve the understanding of the microstructure during recrystallization processes. (C) 2004 Acoustical Society of America. [DOI: 10.1121/1.1810236]
\end{abstract}

PACS numbers: 43.20.Bi, 43.20.Gp, 43.35.Cg [YHB]

Pages: $3319-3327$

\section{INTRODUCTION}

Metals and alloys are made of crystallite grains whose characteristics and arrangements can be changed by the application of heat processing, such as annealing. Microstructural parameters of metals determine the macroscopic mechanical properties of a material and include the grain size, grain shape, and the orientation of the grains, or texture, and their distribution in the microstructure. Ultrasonic waves propagating in such aggregates lose energy due to scattering from the granular microstructure of these materials. This scattering is often characterized by the attenuation of the medium. In general, the attenuation and wave velocity are dependent on the grain size, shape, and on the particular orientation distributions of the grains. If the grains are randomly oriented such that the medium is statistically isotropic, these propagation properties are independent of direction. However, the scattering attenuation and wave velocity are a function of the propagation direction if the grains have a preferred orientation. The preferred orientation of grains, or texture, is best quantitatively described by the orientation distribution function (ODF) defining a probability density function, which is usually expanded in a series of generalized spherical harmonics (Roe, 1965, 1966; Bunge, 1982). Often, most metallic materials with preferred orientation of grains display anisotropy of material properties. Therefore, knowledge of the anisotropic nature of the wave propagation and scattering in textured materials such as attenuation and velocity is critical for use with ultrasonic nondestructive techniques. Such information will provide valuable insight for modeling the microstructure of such complex materials during processing.

The scattering of elastic waves by grains of polycrystals has received considerable attention. The most recent contri-

\footnotetext{
a) Author to whom correspondence should be addressed. Electronic mail: jaturner@unl.edu
}

butions for cubic symmetry with uniformly distributed orientations of grains were made by Hirsekorn (1982, 1983), Stanke (1984), and Weaver (1990). The problem of wave propagation and scattering in the case of polycrystalline grains with an aligned [001] axis has been examined by Ahmed and Thompson (1996) and Turner (1999). In that particular case, the average medium is statistically transversely isotropic. Ahmed and Thompson $(1992,1996)$ also studied correlations defined by both equiaxed grains and grains with elongation.

During the recrystallization process of metals, such as annealing, the microstructure may contain grains having preferred crystallographic orientations. For rolling texture, there are three orthogonal axes of symmetry which are defined as the rolling, transverse, and normal directions. Thus, the material properties of this specific case may be assumed orthorhombic due to the feature of the preferred orientation. Hirsekorn (1985) also was one of the first to investigate the wave scattering in polycrystals of cubic symmetry with rolling texture as a function of frequency by using the perturbation approach. She then extended her theory to determine the directional dependence of the phase velocities and attenuations of the three wave types under the same assumption with fiber texture (Hirsekorn, 1986). Her discussions were restricted to waves propagating in the direction of an axis of symmetry of the texture. The general formalism of the waves propagating in any direction through polycrystalline metals with rolling texture, however, has not yet been reported. The detailed wave velocities of the three wave types, inclusive of the quasilongitudinal and two quasishear waves, have been discussed elsewhere (Sayers, 1982; Johnson, 1985; Hirao et al., 1987; Li and Thompson, 1990) under the assumption of orthorhombic-cubic symmetry.

In this article, the more sensitive ultrasonic parameter, scattering attenuation, is studied for waves propagating in any direction through such textured media. The wave propagation and scattering model is formulated using the Dyson 
equation studied by Frisch (1968) and Weaver (1990) which is easily solved in the spatial Fourier transform domain within the limits of the first-order smoothing approximation (FOSA) or Keller (Karal and Keller, 1964) approximation. The problem is studied here using the anisotropic Green's dyadic, an approach not used previously for textured materials. The attenuations of the three wave types are calculated numerically as a function of dimensionless frequency and propagation direction, respectively, for given orientation distribution coefficients (ODCs) using the derived expressions. The resulting attenuations are shown to be directional dependent, frequency dependent, and dependent on the texture coefficients (ODCs) for the quasilongitudinal and two quasishear waves. The theoretical results presented may be used to improve the understanding of the microstructure during the recrystallization process. In addition, the present formulation may be used to study diffuse ultrasonic problems in a straightforward manner. Although the present model is for the case of orthorhombic-cubic symmetry, the formalism can be easily modified to apply to other given symmetry cases.

In the next section, the preliminary elastodynamics of elastic wave propagation and scattering is introduced in terms of an anisotropic Green's dyadic. The formalism of the attenuation is then developed for the anisotropic case of orthorhombic-cubic symmetry.

\section{WAVE PROPAGATION AND SCATTERING MODEL}

The equation of motion for the elastodynamic response of an infinite, linear-elastic material to deformation is given in terms of the Green's dyadic by

$$
\begin{aligned}
& \left\{-\delta_{j k} \rho \partial_{t}^{2}+\partial x_{i} C_{i j k l}(\mathbf{x}) \partial x_{l}\right\} G_{k \alpha}\left(\mathbf{x}, \mathbf{x}^{\prime} ; t\right) \\
& =\delta_{j \alpha} \delta^{3}\left(\mathbf{x}-\mathbf{x}^{\prime}\right) \delta(t),
\end{aligned}
$$

where $\delta^{3}\left(\mathbf{x}-\mathbf{x}^{\prime}\right)$ is the three-dimensional spatial Delta function. The second-order Green's dyadic, $G_{k \alpha}\left(\mathbf{x}, \mathbf{x}^{\prime} ; t\right)$, defines the response at location $\mathbf{x}$ in the $k$ th direction to a unit impulse at location $\mathbf{x}^{\prime}$ in the $\alpha$ th direction. The moduli are considered to vary spatially and density is assumed uniform throughout. In the case of orthorhombic-cubic symmetry, the moduli $\mathbf{C}$ are supposed to be spatially heterogeneous and have the form $C_{i j k l}(\mathbf{x})=C_{i j k l}^{0}+\delta C_{i j k l}(\mathbf{x})$. The material properties might have global anisotropy such that the mean moduli are not necessarily isotropic. The covariance of the moduli is characterized by an eighth-rank tensor

$$
\left\langle\delta C_{i j k l}(\mathbf{x}) \delta C_{\alpha \beta \gamma \delta}\left(\mathbf{x}^{\prime}\right)\right\rangle=\Xi_{i j k l}^{\alpha \beta \gamma \delta} \eta\left(\mathbf{x}-\mathbf{x}^{\prime}\right) .
$$

The spatial and tensorial parts of the above covariance, $\Xi$ and $\eta$, are assumed independent. The correlation function $\eta$ is also assumed a function of the difference between two vectors, $\mathbf{x}-\mathbf{x}^{\prime}$. This assumption implies that the medium is statistically homogeneous.

The mean response, $\langle\mathbf{G}\rangle$, is governed by the Dyson equation (Weaver, 1990; Frisch, 1968)

$$
\begin{aligned}
\left\langle G_{i \alpha}\left(\mathbf{x}, \mathbf{x}^{\prime}\right)\right\rangle= & G_{i \alpha}^{0}\left(\mathbf{x}, \mathbf{x}^{\prime}\right)+\iint G_{i \beta}^{0}(\mathbf{x}, \mathbf{y}) M_{\beta j}(\mathbf{y}, \mathbf{z}) \\
& \times\left\langle G_{j \alpha}\left(\mathbf{z}, \mathbf{x}^{\prime}\right)\right\rangle d^{3} y d^{3} z .
\end{aligned}
$$

In Eq. (3), the quantity $\mathbf{G}^{0}$ is the bare Green's dyadic defined as the ensemble average response of the medium without fluctuations, namely, the solution to Eq. (1) when $\delta C_{i j k l}(\mathbf{x})$ $=0$. The second order tensor $\mathbf{M}$ is the mass or self-energy operator. The Dyson equation, Eq. (3), is easily solved in the Fourier transform domain under the assumption of statistical homogeneity. The assumption of statistical homogeneity ensures that $\mathbf{G}^{0}, \mathbf{M}$, and $\langle\mathbf{G}\rangle$ are functions of a single wave vector in Fourier space. The Dyson equation is then transformed and solved to give the result for $\langle\mathbf{G}(\mathbf{p})\rangle$ of the form

$$
\langle\mathbf{G}(\mathbf{p})\rangle=\left[\mathbf{G}^{0}(\mathbf{p})^{-1}-\tilde{\mathbf{M}}(\mathbf{p})\right]^{-1},
$$

where $\tilde{\mathbf{M}}$ is the spatial transform of the self-energy. The selfenergy $\mathbf{M}$ can be written as an expansion in powers of moduli fluctuations. To first order (Frisch, 1968; Karal and Keller, 1964) M is expressed as (Weaver, 1990)

$$
\begin{aligned}
& M_{\beta j}(\mathbf{y}, \mathbf{z}) \\
& \quad \approx\left\langle\frac{\partial}{\partial y_{\alpha}} \delta C_{\alpha \beta \gamma \delta}(\mathbf{y}) \frac{\partial}{\partial y_{\delta}} G_{\gamma k}^{0}(\mathbf{y}, \mathbf{z}) \frac{\partial}{\partial z_{i}} \delta C_{i j k l}(\mathbf{z}) \frac{\partial}{\partial z_{l}}\right\rangle .
\end{aligned}
$$

Such an approximation is assumed valid if the fluctuations, $\delta \mathbf{C}$, are not too large. The components of $\widetilde{\mathbf{M}}$ are employed to calculate the attenuation of the three wave modes. Further details of the scattering theory can be reviewed by the reader in the articles of Karal and Keller (1964), Frisch (1968), Stanke and Kino (1984), Weaver (1990), and Turner (1999).

The medium of oriented grains with rolling texture has orthorhombic symmetry. When ultrasonic waves propagate in such media, the phase velocity and the associated polarization vector are determined by the Christoffel equation. The dispersion relations for the mean response are then given by the solution of the Dyson equation, Eq. (4), as

$$
\begin{aligned}
g_{\beta}(\mathbf{p}) & =\left[g_{\beta}^{0}(\mathbf{p})^{-1}-m_{\beta}(\mathbf{p})\right]^{-1} \\
& =\left[\omega^{2}-p^{2} c_{\beta}^{2}-m_{\beta}(\mathbf{p})\right]^{-1},
\end{aligned}
$$

for each wave type, $\beta$, quasilongitudinal $(q P)$ and two quasishear ( $q S 1$ and $q S 2)$ waves. The expressions for the dispersion relations of the mean response are written

$$
\omega^{2}-p^{2} c_{\beta}^{2}-m_{\beta}(\mathbf{p})=0,
$$

which is solved for the wave vector $\mathbf{p}$. The attenuation of each wave type is given by the imaginary part of $\mathbf{p}$. The explicit expressions of the attenuation can be determined using an approximation valid below the high-frequency geometric optics limit $\left[m_{\beta}(\mathbf{p}) \approx m_{\beta}\left(\left(\omega / c_{\beta}\right) \hat{\mathbf{p}}\right)\right]$ (Stanke and Kino, 1984; Weaver, 1990). This approximation allows the imaginary part of $\mathbf{p}$ to be calculated directly from Eq. (7). Thus, the attenuations of the three wave types are calculated as

$$
\alpha_{\beta}(\hat{\mathbf{p}})=-\frac{1}{2 \omega c_{\beta}(\hat{\mathbf{p}})} \operatorname{Im} m_{\beta}\left(\frac{\omega}{c_{\beta}} \hat{\mathbf{p}}\right) .
$$

The attenuations for the three wave types, which are each defined in Eq. (8), are finally given in the general form (Turner, 1999) 


$$
\begin{aligned}
& \alpha_{\beta}(\hat{\mathbf{p}})=\frac{1}{c_{\beta}^{3}(\hat{\mathbf{p}})}\left\{\frac { \pi } { 4 } \int d ^ { 2 } \hat { s } \frac { \omega ^ { 4 } } { c _ { q S 1 } ^ { 5 } ( \hat { \mathbf { s } } ) } \tilde { \eta } \left(\frac{\omega}{c_{\beta}(\hat{\mathbf{p}})} \hat{\mathbf{p}}\right.\right. \\
& \left.-\frac{\omega}{c_{q S 1}(\hat{\mathbf{s}})} \hat{\mathbf{s}}\right)_{\cdots \hat{\mathbf{u}}_{K} \hat{\mathbf{p}} \hat{\mathbf{v}}_{1}}^{\cdots \hat{\mathbf{u}}_{K} \hat{\mathbf{p}} \hat{\mathbf{v}}_{1}} \\
& +\frac{\pi}{4} \int d^{2} \hat{s} \frac{\omega^{4}}{c_{q P}^{5}(\hat{\mathbf{s}})} \tilde{\eta}\left(\frac{\omega}{c_{\beta}(\hat{\mathbf{p}})} \hat{\mathbf{p}}\right. \\
& \left.-\frac{\omega}{c_{q P}(\hat{\mathbf{s}})} \hat{\mathbf{s}}\right) \Xi \begin{array}{l}
\ldots \hat{\mathbf{u}}_{K} \hat{\mathbf{p}} \hat{\mathbf{s}} \hat{\mathbf{v}}_{2} \\
\ldots \hat{\mathbf{u}}_{K} \hat{\mathbf{p}} \hat{\mathbf{s}}_{2}
\end{array} \\
& +\frac{\pi}{4} \int d^{2} \hat{s} \frac{\omega^{4}}{c_{q S 2}^{5}(\hat{\mathbf{s}})} \tilde{\eta}\left(\frac{\omega}{c_{\beta}(\hat{\mathbf{p}})} \hat{\mathbf{p}}\right.
\end{aligned}
$$

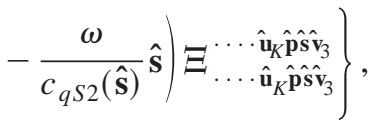

where $K$ is defined as the polarization for the wave type $\beta$ (1, 2 , or 3 for wave types $q S 1, q P$, and $q S 2$, respectively). In the above equation, the integrals are over the unit sphere, which is defined by unit vector $\hat{\mathbf{s}}$. The direction $\hat{\mathbf{p}}$ defines the propagation direction, $\hat{\mathbf{s}}$ is the scattered direction, and $\hat{\mathbf{u}}$ and $\hat{\mathbf{v}}$ are defined as the polarization directions. The dependence of the vectors $\hat{\mathbf{u}}$ on $\hat{\mathbf{p}}$ and of $\hat{\mathbf{v}}$ on $\hat{\mathbf{s}}$ is implicit. The argument of $\tilde{\eta}$ is the difference between the incoming and outgoing propagation directions. The inner products on the covariance of the moduli fluctuations are given in terms of four unit vectors. In the next section, the correlation function is specified.

\section{CORRELATION FUNCTION}

As shown in Eq. (2), the tensorial and spatial contributions of the material covariance are assumed independent. The spatial correlations are characterized by $\eta$. Here, it is assumed that $\eta$ has an exponential form

$$
\eta(\mathbf{r})=e^{-r / L} \text {. }
$$

The correlation length, $L$, is of the order of the grain radius in polycrystals. In general, a simple exponential form of the spatial correlation function is not exact for polycrystals with rolling texture for which there is grain elongation. For elongated grains, a more general spatial correlation function must be used (Ahmed and Thompson, 1992). The influence of this choice of correlation function on the attenuations is left as a subject of future investigations. In Fourier transform space, the correlation function is then given by

$$
\tilde{\eta}(\mathbf{q})=\frac{L^{3}}{\pi^{2}\left(1+L^{2} q^{2}\right)^{2}} .
$$

The forms of the attenuation given above contain the difference of two vectors, $\tilde{\eta}(\mathbf{q})=\tilde{\eta}\left(\left[\omega / c_{1}(\theta)\right] \hat{\mathbf{p}}\right.$ $\left.-\left[\omega / c_{2}\left(\theta^{\prime}\right)\right] \hat{\mathbf{s}}\right)$ as the argument for covariance in Eq. (2). Now the correlation functions $\tilde{\eta}_{\beta-\gamma}(\hat{\mathbf{p}}, \hat{\mathbf{s}})$ are considered. If the three nondimensional frequencies are then defined as $x_{\beta}=\omega L / c_{\beta}$, using the expression of the spatial Fourier transform of the correlation function in Eq. (11), the func- tions $\tilde{\eta}_{\beta-\gamma}(\hat{\mathbf{p}}, \hat{\mathbf{s}})$ are then expressed in terms of the above dimensionless quantities as

$$
\tilde{\eta}_{\beta-\gamma}(\hat{\mathbf{p}}, \hat{\mathbf{s}})=\frac{L^{3}}{\pi^{2}\left(1+x_{\beta}^{2}(\phi)+x_{\gamma}^{2}\left(\phi^{\prime}\right)-2 x_{\beta}(\phi) x_{\gamma}\left(\phi^{\prime}\right) \hat{\mathbf{p}} \cdot \hat{\mathbf{s}}\right)^{2}},
$$

for the incoming wave type $\beta$ and outgoing wave type $\gamma$. The inner product, $\quad \hat{\mathbf{p}} \cdot \hat{\mathbf{s}}=\cos \phi \cos \phi^{\prime} \sin \theta \sin \theta^{\prime}$ $+\sin \phi \sin \phi^{\prime} \sin \theta \sin \theta^{\prime}+\cos \theta \cos \theta^{\prime}$, if the unit vectors $\hat{\mathbf{p}}$ and $\hat{\mathbf{s}}$ are generally defined by $\hat{\mathbf{p}}=\mathbf{x}_{1} \cos \phi \sin \theta$ $+\mathbf{x}_{2} \sin \phi \sin \theta+\mathbf{x}_{3} \cos \theta \quad$ and $\hat{\mathbf{s}}=x_{1} \cos \phi^{\prime} \sin \theta^{\prime}$ $+\mathbf{x}_{2} \sin \phi^{\prime} \sin \theta^{\prime}+\mathbf{x}_{3} \cos \theta^{\prime}$. The angles $\theta, \phi$ and $\theta^{\prime}, \phi^{\prime}$ are respectively defined as Euler angles in a general coordinate system. The form of the eighth-rank tensor, $\Xi_{i j k l}^{\alpha \beta \gamma \delta}$, is discussed next for rolling texture made up of cubic crystallites.

\section{COVARIANCE AND ATTENUATION}

To calculate the attenuations, the relevant inner products on the covariance of the moduli fluctuations are required. The covariance of the moduli fluctuations is represented by an eighth-rank tensor which is given explicitly by

$$
\Xi_{\cdots \cdots \hat{\mathbf{u}} \hat{\mathbf{p}} \hat{\mathbf{s}} \hat{\mathbf{v}}}=\Xi_{\alpha \beta \gamma \delta}^{i j k l} \hat{u}_{\beta} \hat{u}_{k} \hat{p}_{\alpha} \hat{p}_{l} \hat{s}_{i} \hat{s}_{\delta} \hat{v}_{\gamma} \hat{v}_{j} .
$$

For polycrystals of cubic symmetry, the eighth-rank covariance, $\Xi_{i j k l}^{\alpha \beta \gamma \delta}$, is written as

$$
\begin{aligned}
\Xi_{i j k l}^{\alpha \beta \gamma \delta}= & \left\langle C_{i j k l} C_{\alpha \beta \gamma \delta}\right\rangle-\left\langle C_{i j k l}\right\rangle\left\langle C_{\alpha \beta \gamma \delta}\right\rangle \\
= & \kappa^{2}\left\langle\sum_{n=1}^{3} a_{i n} a_{j n} a_{k n} a_{l n} \sum_{n=1}^{3} a_{\alpha n} a_{\beta n} a_{\gamma n} a_{\delta n}\right\rangle \\
& -\kappa^{2}\left\langle\sum_{n=1}^{3} a_{i n} a_{j n} a_{k n} a_{l n}\right\rangle \\
& -\left\langle\sum_{n=1}^{3} a_{\alpha n} a_{\beta n} a_{\gamma n} a_{\delta n}\right\rangle
\end{aligned}
$$

where the brackets, \langle\rangle , denote an ensemble average over all orientations of grains, and $\kappa=C_{11}^{0}-C_{12}^{0}-2 C_{44}^{0}$ is the singlecrystal anisotropy factor. If the polycrystal is of orthorhombic-cubic symmetry, only certain terms are nonzero. An example term necessary for calculating the attenuations is presented in the Appendix. Details of the other nonzero terms may be found elsewhere (Yang, 2003). For the second term in Eq. (14), the results are given in the details of other articles (Sayers, 1982; Johnson, 1985; Hirao et al., 1987; Li and Thompson, 1990).

The forms of the attenuations presented in Eq. (9) require various inner products on the covariance tensor. These inner products have the general form of $\Xi \ldots . \hat{\text { ûp } \hat{p} \hat{\mathbf{v}} \hat{\mathbf{v}} \hat{\mathbf{v}}}$, where the vectors $\hat{\mathbf{p}}$ and $\hat{\mathbf{s}}$, respectively, represent the incoming and outgoing propagation directions. The vectors $\hat{\mathbf{u}}$ and $\hat{\mathbf{v}}$ are vectors defining the polarization directions of the particular waves. While waves propagate in arbitrary directions, the polarization vectors are found by the Christoffel equation. Substituting the correlation function, Eq. (12), and the inner products into Eq. (9), the resulting dimensionless attenuations are given in the form 


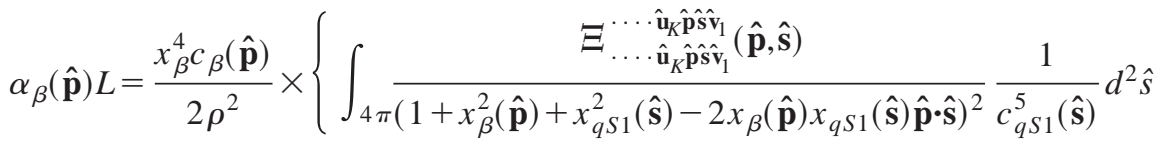

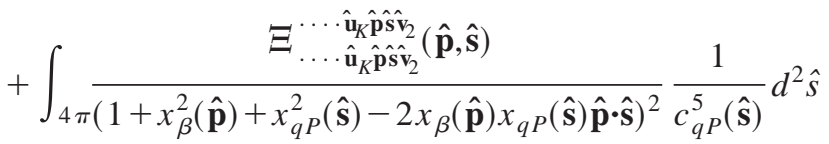

$$
\begin{aligned}
& +\int_{4 \pi\left(1+x_{\beta}^{2}(\hat{\mathbf{p}})+x_{q S 2}^{2}(\hat{\mathbf{s}})-2 x_{\beta}(\hat{\mathbf{p}}) x_{q S 2}(\hat{\mathbf{s}}) \hat{\mathbf{p}} \cdot \hat{\mathbf{s}}\right)^{2}} \frac{1}{c_{q S 2}^{5}(\hat{\mathbf{s}})} d^{2} \hat{\mathbf{u}_{K} \hat{\mathbf{p}} \hat{\mathbf{s}} \hat{\mathbf{v}}_{3}}(\hat{\mathbf{s}}\},
\end{aligned}
$$

where $K$ has the same definition as discussed in Eq. (9). It should be noted that these inner products have units of $\kappa^{2}$. In the long wavelength Rayleigh limit, $x_{\beta} \ll 1$, Eq. (15) can be simplified as

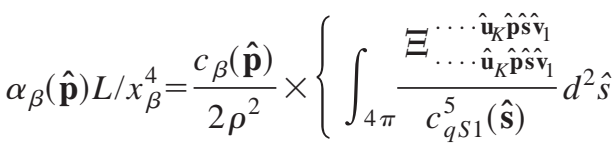

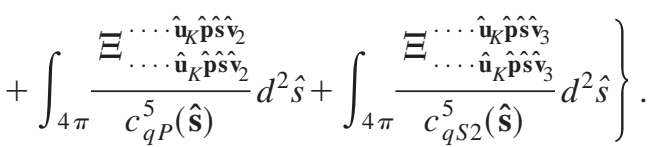

In Eq. (16), the dimensionless attenuation has been normalized by the fourth power of the dimensionless frequency for the respective wave type.

In Eqs. (15) and (16) the inner products, $\Xi \cdots$. not have simple analytical forms for arbitrary propagation direction in this orthorhombic-cubic case. Thus, these results must be calculated numerically. In the next section, example numerical results and discussions are presented.

\section{NUMERICAL RESULTS AND DISCUSSIONS}

Numerical results are now presented for a $70 \%$ rolled steel plate. The material constants of a single crystal and the texture coefficients of the orientation distribution function with respect to the generalized spherical functions are given by (Bunge, 1982)

$$
\begin{aligned}
& C_{11}^{0}=2.37 \times 10^{11} \mathrm{~Pa}, \quad C_{12}^{0}=1.41 \times 10^{11} \mathrm{~Pa}, \\
& C_{44}^{0}=1.16 \times 10^{11} \mathrm{~Pa}, \quad \rho=7850 \mathrm{~kg} / \mathrm{m}^{3},
\end{aligned}
$$

and

$$
\begin{aligned}
& c_{4}^{00}=-1.47, \quad c_{4}^{20}=0.46, \\
& c_{4}^{40}=0.50, \quad c_{6}^{00}=2.69, \\
& c_{6}^{20}=-1.20, \quad c_{6}^{40}=0.46, \\
& c_{6}^{60}=-0.14, \quad c_{8}^{00}=-0.07, \\
& c_{8}^{20}=0.29, \quad c_{8}^{40}=-0.45, \\
& c_{8}^{60}=-0.47, \quad c_{8}^{80}=-0.22 .
\end{aligned}
$$

The orientation distribution coefficients (ODCs) in Bunge's notation $c_{l}^{m n}$ must be converted into those in Roe's notation $W_{l m n}$, which are used in this discussion. In order to carry out the calculations for the attenuations, Eq. (15), numerical methods are employed. The procedure of numerical methods for calculating the wave attenuations is now described.

First, using the Christoffel equation, the eigenvalueeigenvector problem is solved for a given wave propagation direction and scattering direction. Second, the covariance of the moduli fluctuations is calculated by Eq. (14). Next, using the known covariance and eigenvectors, the inner products of each wave type are calculated numerically. Finally, the double integration is implemented numerically by the extended trapezoidal method. Here, examples are presented to describe important features of the wave attenuations for several propagation directions. The examples are generated using the methods discussed above. Since the orthorhombic symmetry has three mutually orthogonal planes of symmetry, all calculations are made for $0^{\circ} \leqslant \phi \leqslant 90^{\circ}$ and $0^{\circ} \leqslant \theta \leqslant 90^{\circ}$.

Convergence of the numerical integration was examined first. Wave attenuations of each wave type were examined for waves propagating in the rolling direction, that is $\phi=0^{\circ}$ and $\theta=90^{\circ}$, and at a dimensionless frequency $x_{q S 1}=1.0$. The results show fast convergence for each wave mode in numerical integrations using the extended trapezoidal method (Yang, 2003). In order to achieve a balance between efficiency and accuracy, the number of intervals in the integra-

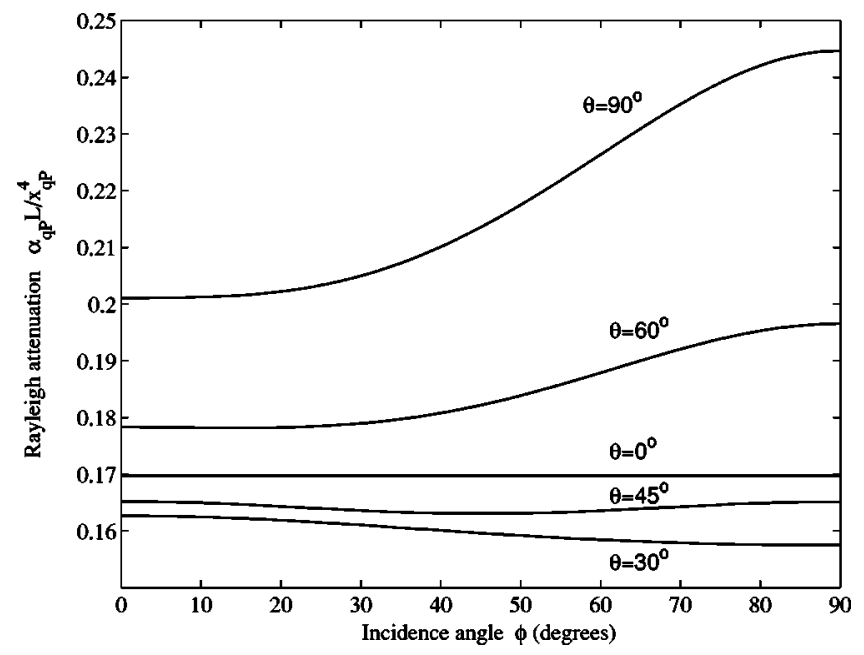

FIG. 1. Rayleigh attenuation, $\alpha_{q P} L / x_{q P}^{4}$, as a function of propagation direction for $q P$ waves using the specified ODCs. 


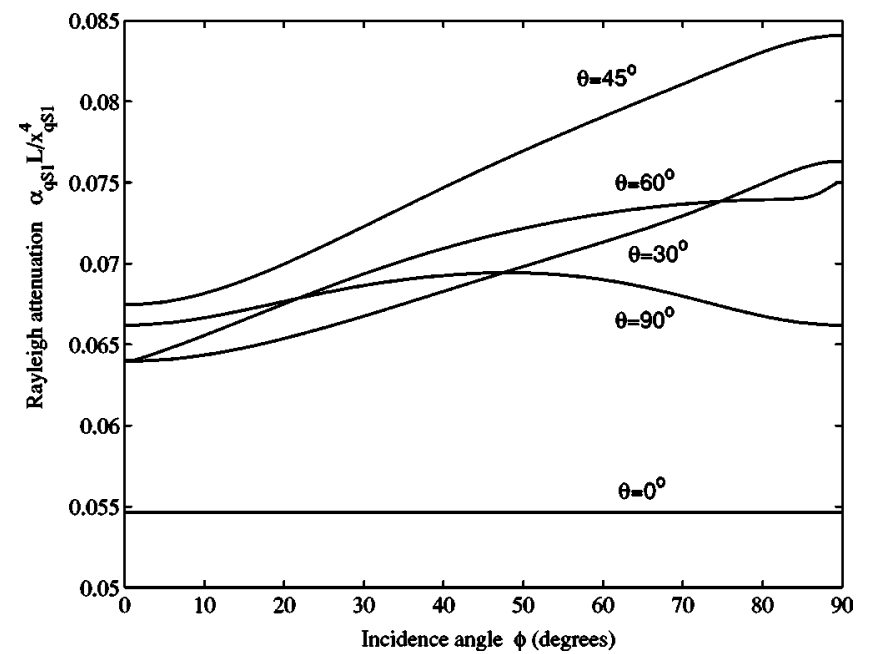

FIG. 2. Rayleigh attenuation, $\alpha_{q S 1} L / x_{q S 1}^{4}$, as a function of propagation direction for $q S 1$ waves using the specified ODCs.

tion $N=20$ is chosen for calculating the attenuations for each wave mode in the results shown here.

First, the attenuations within the Rayleigh limit are calculated using Eq. (16). It is known that the attenuations depend on the fourth power of frequency in the Rayleigh regime. Thus, the normalized Rayleigh attenuation $\alpha_{\beta} L / x_{\beta}^{4}$ of each wave mode is shown with the angular dependence in Figs. 1-3 for various propagation directions, respectively. It is observed that in this specific case the attenuations of each wave mode are considerably dependent on the wave propagation direction. For waves propagating in different directions, the curves of the attenuations have smoothly changing shapes. The percent variations of the $q P, q S 1$, and $q S 2$ attenuations in terms of polar angle $\theta$ shown in Figs. 1-3 are about $25-40 \%, 20-50 \%$, and $15 \%$, respectively. The variation of the $q S 2$ attenuations is more uniform than that of the others.

Outside the Rayleigh regime, the attenuation results are calculated using the complete integrals, Eq. (15). The directional dependence of the attenuation is presented first for a given dimensionless frequency, $x_{q S 1}=1.0$. Figure 4 shows

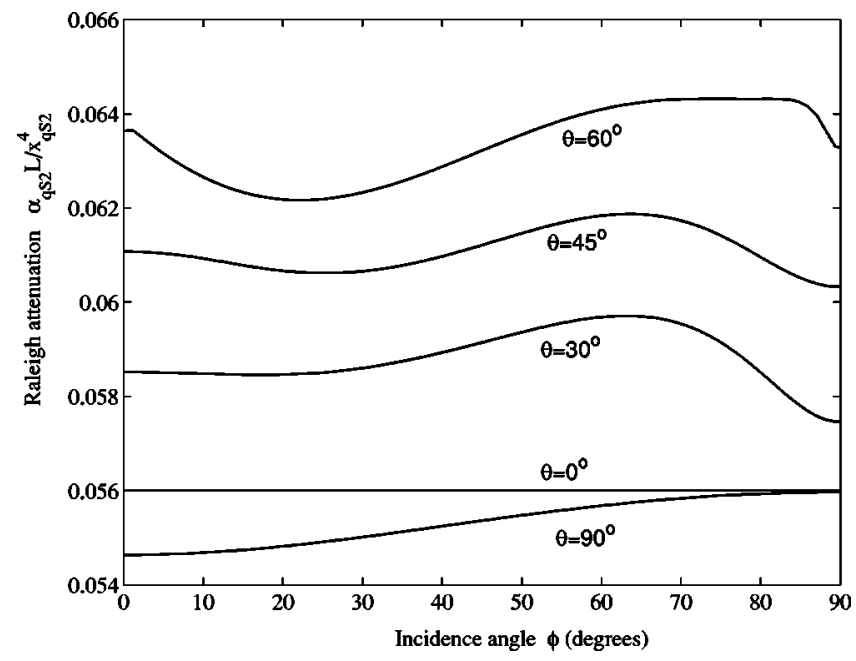

FIG. 3. Rayleigh attenuation, $\alpha_{q S 2} L / x_{q S 2}^{4}$, as a function of propagation direction for $q S 2$ waves using the specified ODCs.

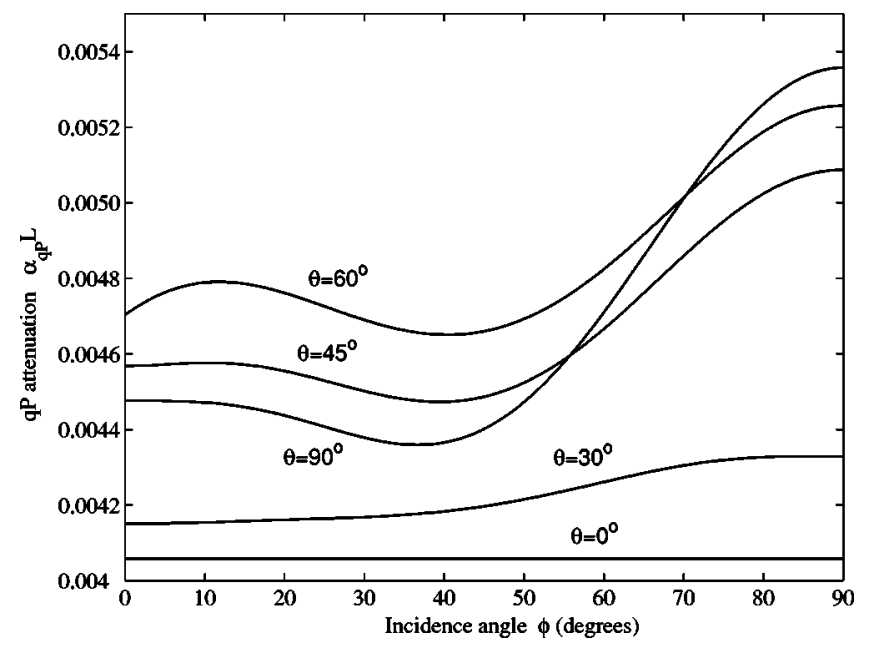

FIG. 4. Directional dependence of the normalized $q P$ attenuation, $\alpha_{q P} L$, for frequency $x_{q S 1}=1.0$.

the normalized quasilongitudinal wave $(q P)$ attenuation, $\alpha_{q P} L$, as a function of azimuthal direction $\phi$ for various polar angles $\theta$. It is seen that the attenuation is dependent on the propagation direction as expected. Here, the attenuation variation with respect to polar angle $\theta$ is around $15-30 \%$. The results for the normalized shear wave $(q S 1$ and $q S 2)$ attenuations are presented in Figs. 5 and 6, respectively. The directional dependence on the propagation direction for these attenuations is also significant. The percent variation of the $q S 1$ and $q S 2$ attenuations in terms of polar angle $\theta$ is about $10-50 \%$. These results may be contrasted with the results in the Rayleigh limit. Comparisons of the Rayleigh attenuations with attenuations outside the Rayleigh regime show that the tendency of variation is quite different with each due to the effect of frequency. In Fig. 4, the $q P$ wave attenuation is observed to have the maximum at $\phi=90^{\circ}$ for given angles $\theta$. In Fig. 5, the curves of the $q S 1$ wave attenuations have smoothly changing shapes. Figure 6 shows that for propagation at polar angle $\theta=30^{\circ}, 45^{\circ}$, and $60^{\circ}$, the maximum attenuation is about $\phi=45^{\circ}$, and at polar angle $\theta=90^{\circ}$, there is a minimum attenuation approximately at $\phi=45^{\circ}$. Further-

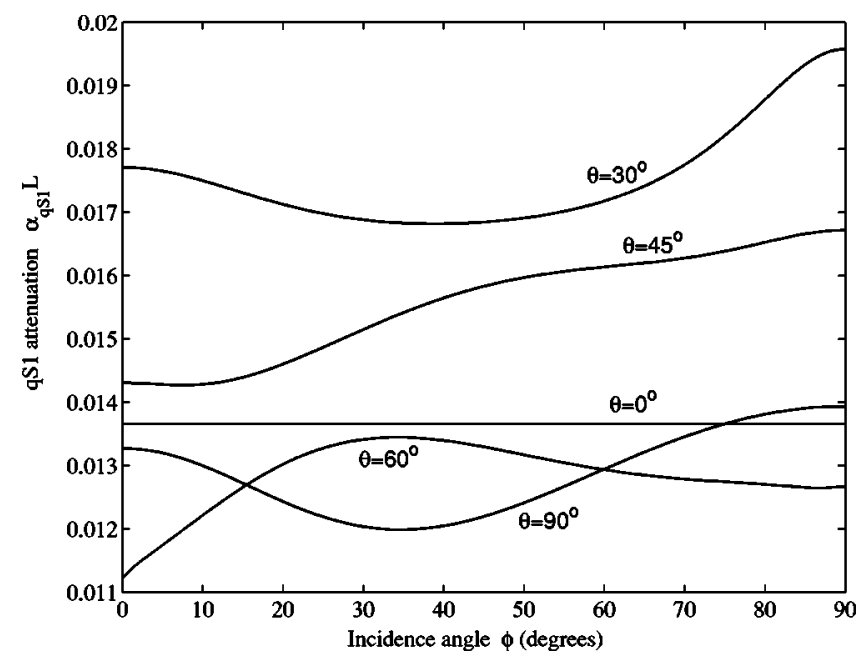

FIG. 5. Directional dependence of the normalized $q S 1$ attenuation, $\alpha_{q S 1} L$, for frequency $x_{q S 1}=1.0$. 


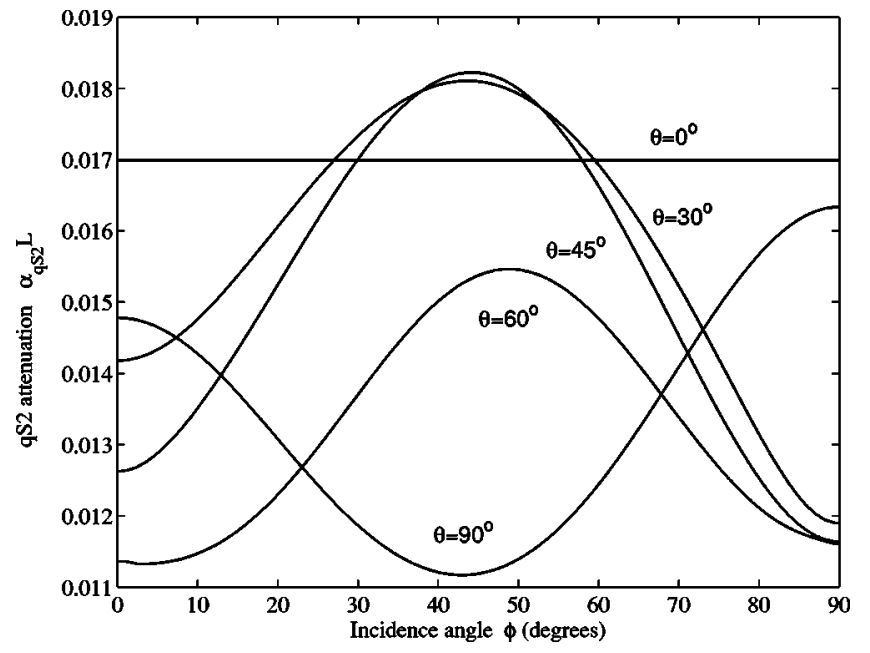

FIG. 6. Directional dependence of the normalized $q S 2$ attenuation, $\alpha_{q S 2} L$, for frequency $x_{q S 1}=1.0$.

more, the asymmetry is observed in Fig. 6 for various polar angles.

Next, results are presented for the normalized attenuation as a function of aximuthal direction $\phi$ for four different frequencies at given polar angle $\theta=90^{\circ}$. The normalized shear wave $(q S 1)$ attenuations, $\alpha_{q S 1} L$, are shown in Fig. 7 for $\theta=90^{\circ}$ for normalized frequencies $x_{q S 1}=1.0,1.5,2.0$, and 2.5. It is seen that the attenuation curves show a similar shape with increasing frequency for the respective polar angle. The results for the normalized $q P$ attenuations, $\alpha_{q P} L$, are shown in Fig. 8 for the same frequencies. Figure 9 shows the normalized $q S 2$ attenuations, $\alpha_{q S 2} L$, as a function of propagation direction for the same four frequencies. It is observed that the attenuation curves show a similar shape with increasing frequency for each wave type as well. All curves of the attenuations have smoothly changing shapes for various frequencies. Figure 9 shows that at polar angle $\theta=90^{\circ}$ there is a minimum attenuation at $\phi=45^{\circ}$ for various frequencies. There is no symmetry to be observed with increasing frequency as well in Fig. 9.

Finally, results are presented for the normalized attenu-

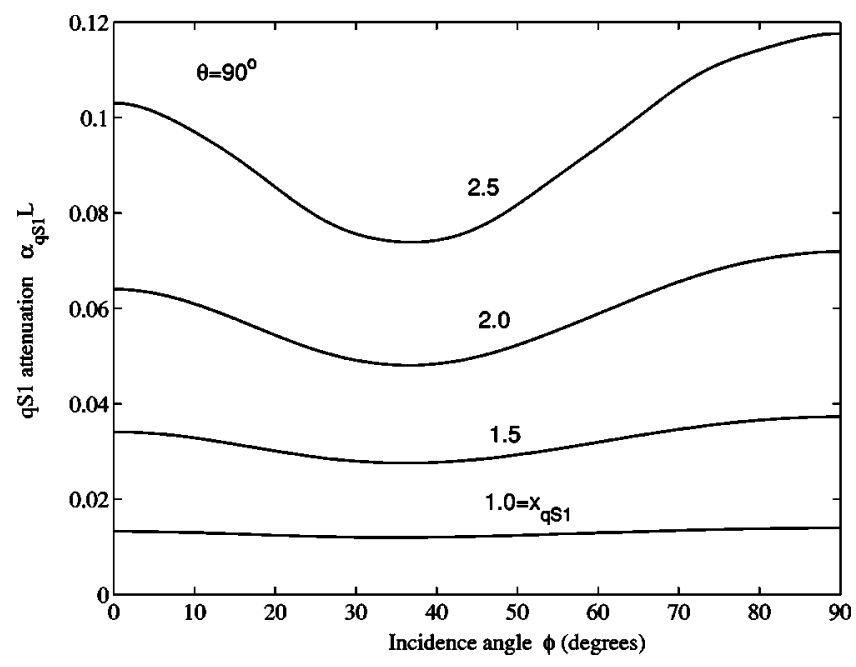

FIG. 7. Angular dependence of the normalized $q S 1$ attenuation, $\alpha_{q S 1} L$, for various frequencies, $x_{q S 1}$, at polar angle $\theta=90^{\circ}$.

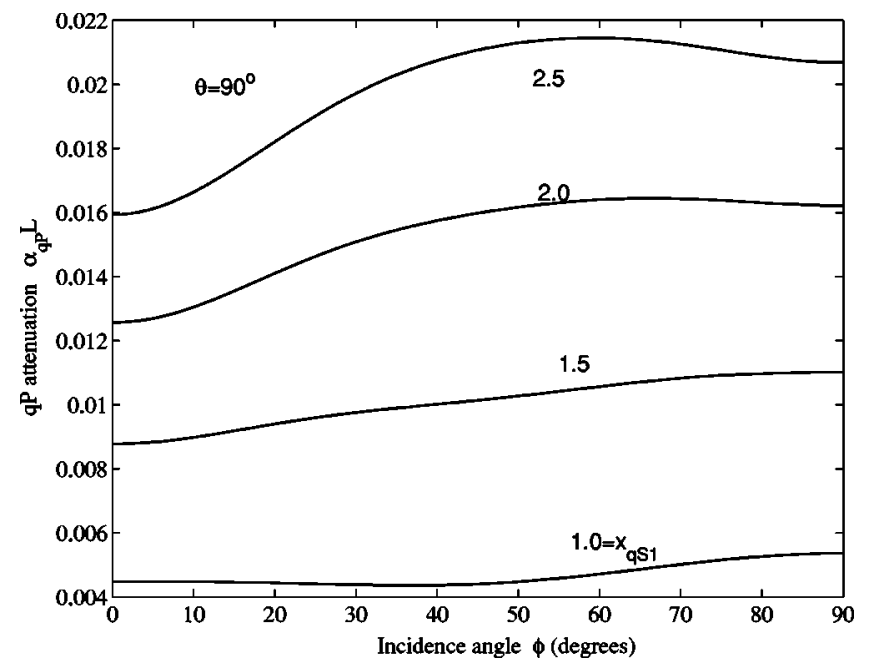

FIG. 8. Angular dependence of the normalized $q P$ attenuation, $\alpha_{q P} L$, for various frequencies, $x_{q S 1}$, at polar angle $\theta=90^{\circ}$.

ations as a function of frequency for several propagation directions. In Figs. 10-12, the normalized attenuations of the three wave modes are plotted versus dimensionless frequency, $x_{q S 1}$, for propagation directions along the rolling, normal, and transverse directions, respectively. For the example considered here, Fig. 11 shows the $q S 1$ wave attenuation for propagation in the rolling, normal, and transverse directions with polarization in the normal, transverse, and rolling directions, respectively. Figure 12 shows the $q S 2$ wave attenuation propagated in the rolling, normal, and transverse directions and polarized in the transverse, rolling, and normal directions, respectively. It is observed that there is a transition region as the dimensionless frequency increases. Thus, the relative order of the attenuation is switched in such a transition region for the three wave modes, respectively. The attenuations increase with the fourth power of frequency in the low frequency limit. After a transition region, the attenuations scale with the square of frequency as expected. Moreover, the normalized attenuation of each wave type is plotted versus normalized frequency, $x_{q S 1}$, for propagation within the $x_{1}-x_{2}$ plane for various

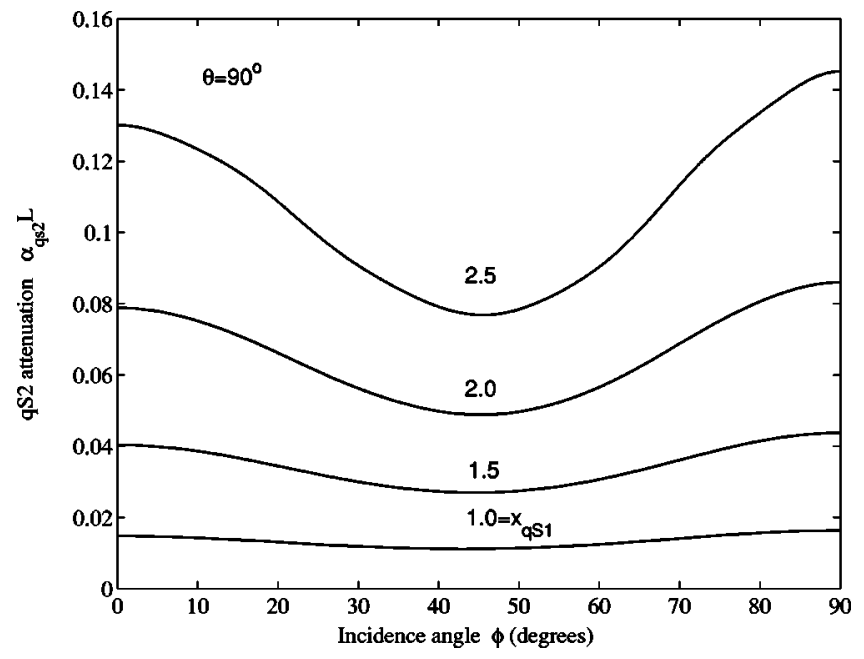

FIG. 9. Angular dependence of the normalized $q S 2$ attenuation, $\alpha_{q S 2} L$, for various frequencies, $x_{q S 1}$, at polar angle $\theta=90^{\circ}$.

L. Yang and J. A. Turner: Scattering in polycrystals with texture 


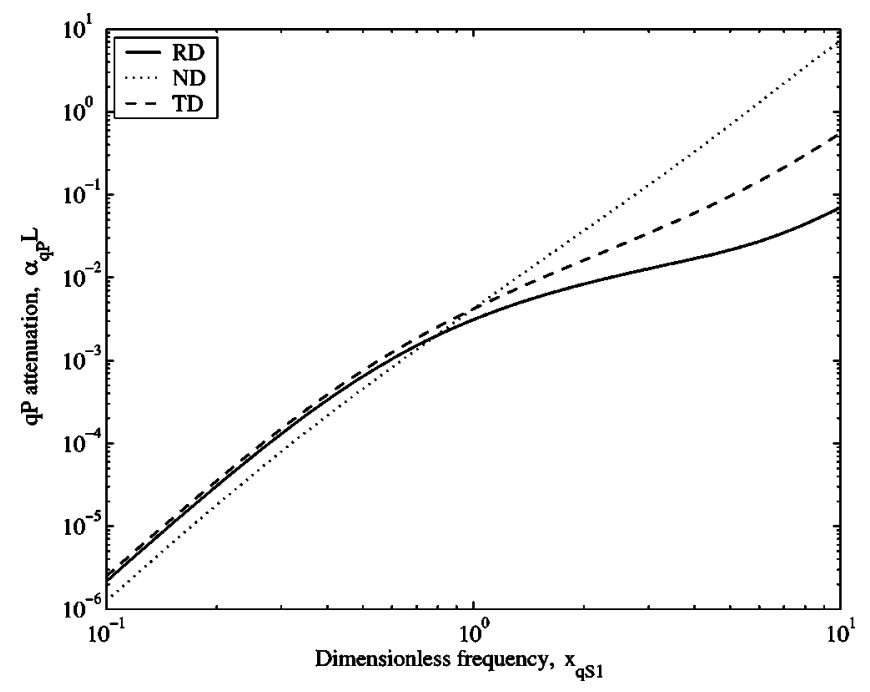

FIG. 10. Normalized $q P$ attenuation, $\alpha_{q P} L$, as a function of dimensionless frequency, $x_{q S 1}$, for waves propagating in the rolling $(R D)$, normal $(N D)$ and transverse $(T D)$ directions.

azimuthal angles in Figs. 13-15. In the Rayleigh regime, it is observed that the curves for each wave type mainly show the same shape. The variation of the attenuation of each wave mode is about $10 \%$. Outside the Rayleigh regime, the attenuation of each wave mode in the rolling direction is about ten times higher than that for propagation at $\phi=30^{\circ}, 45^{\circ}$, and $60^{\circ}$. This feature is thought to be the result of the weak texture for the case discussed here.

The generalized attenuation results presented in this article for arbitrary propagation direction suggest that new ultrasonic techniques for characterization of texture coefficients may be possible. Further study is necessary to unravel the complex relations between the ODCs and the angular and frequency dependence of the attenuations. Attenuation measurements could ultimately be inverted such that the ODCs may be determined. However, such an approach must be optimized by choosing measurement directions that are the most sensitive to the desired ODC. Thus, theoretical devel-

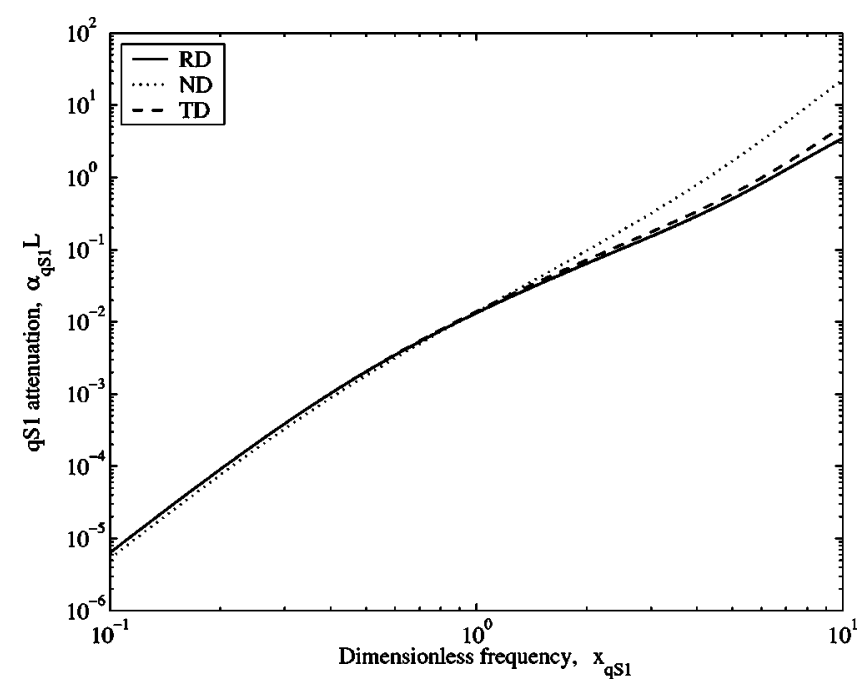

FIG. 11. Normalized $q S 1$ attenuation, $\alpha_{q S 1} L$, as a function of dimensionless frequency, $x_{q S 1}$, for waves propagating in the rolling $(R D)$, normal $(N D)$ and transverse $(T D)$ directions.

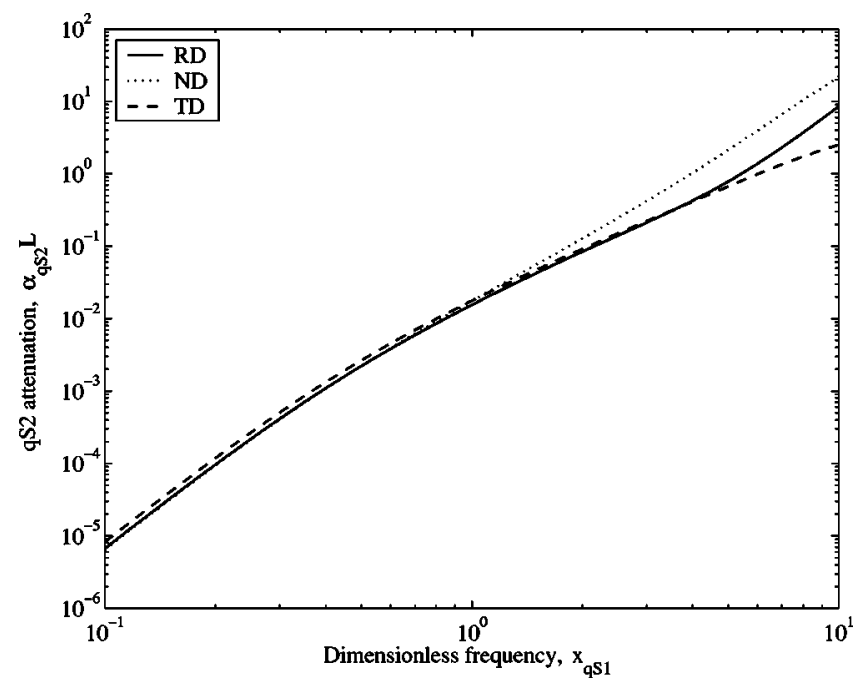

FIG. 12. Normalized $q S 2$ attenuation, $\alpha_{q S 2} L$, as a function of dimensionless frequency, $x_{q S 1}$, for waves propagating in the rolling $(R D)$, normal $(N D)$ and transverse $(T D)$ directions.

opments such as this one will provide a firm basis for directing new experiments. Eventual process control of recrystallization that is quantitative will require modeling-directed experimental methods. In addition to theoretical research, numerical methods will also be necessary for progress to be made. This work must also be expanded to include other factors important to textured materials, such as grain size distribution and grain shape.

\section{SUMMARY}

In this article, the scattering of elastic waves in polycrystalline materials with texture was discussed. The ensemble average response of the elastic waves is governed by the Dyson equation within the limits of first-order smoothing approximation. In contrast with previous work, here an anisotropic Green's dyadic approach was used. In order to calculate the attenuations, the relevant inner products on the covariance of the effective moduli fluctuations were derived

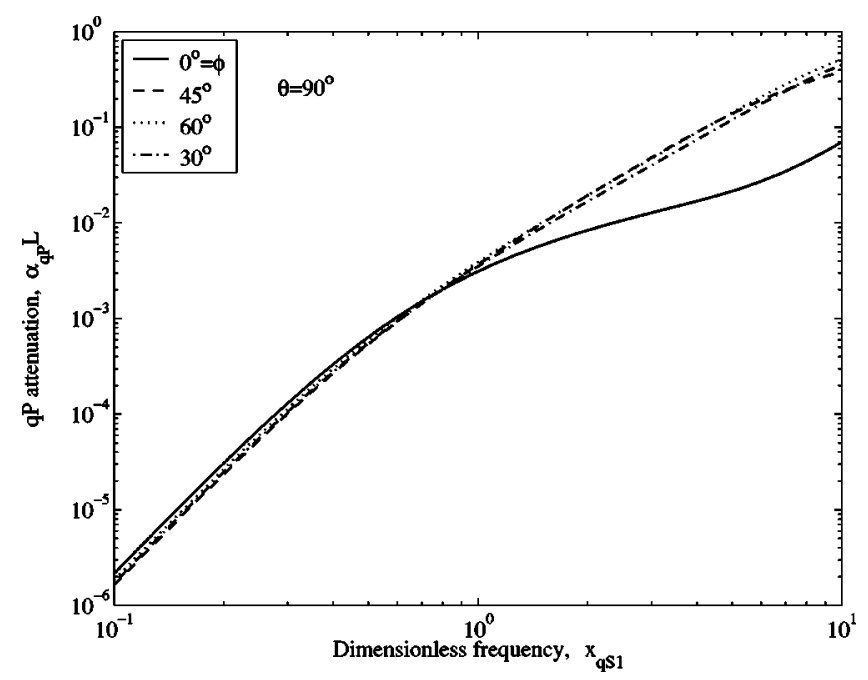

FIG. 13. Normalized $q P$ attenuation, $\alpha_{q P} L$, as a function of dimensionless frequency, $x_{q S 1}$, for waves propagating within the $x_{1}-x_{2}$ plane. 


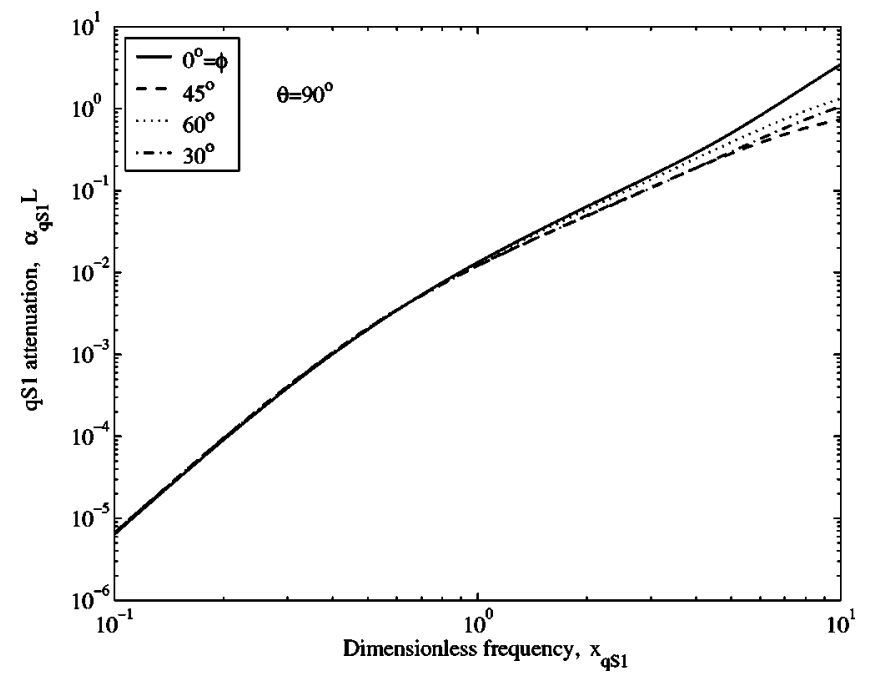

FIG. 14. Normalized $q S 1$ attenuation, $\alpha_{q S 1} L$, as a function of dimensionless frequency, $x_{q S 1}$, for waves propagating within the $x_{1}-x_{2}$ plane.

in polycrystals of cubic symmetry with rolling texture. Compact expressions for the attenuations of the quasilongitudinal and two quasishear waves were then presented in terms of integrations over the scattered directions. In general, attenuations for each wave type are dependent on frequency, wave velocity, and wave propagation direction, as well as texture coefficients which are the expansion coefficients of the orientation function with respect to the generalized spherical functions. The results show that the attenuations of each wave type can be comprehensively affected by those parameters. The general formulation is also directly related to backscattering problems. The simple form of the results makes them particularly useful for nondestructive testing and materials characterization research. To use ultrasonic techniques for monitoring texture during processing, the relation-

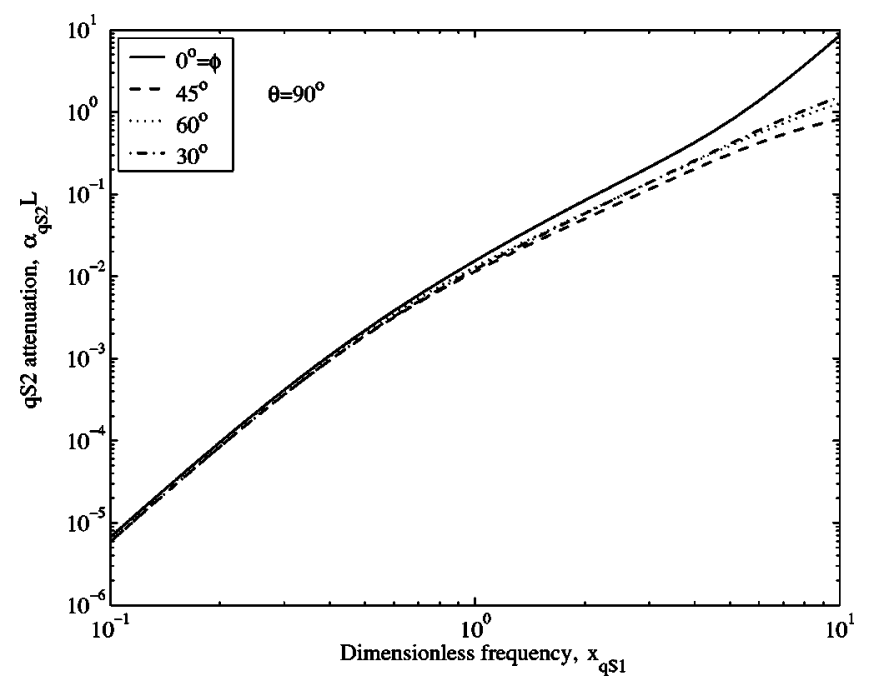

FIG. 15. Normalized $q S 2$ attenuation, $\alpha_{q S 2} L$, as a function of dimensionless frequency, $x_{q S 1}$, for waves propagating within the $x_{1}-x_{2}$ plane.

ships between ultrasonic parameters such as ultrasonic attenuation and materials texture must be investigated. If one knows the relationships between the ODCs and the ultrasonic attenuation, the texture coefficients can be inverted from ultrasonic attenuation measurements. The ultrasonic attenuations of sample specimens can then be measured during annealing such that the ODCs can be determined during processing.

\section{ACKNOWLEDGMENTS}

The financial support of the U.S. Department of Energy (Grant No. DE-FG02-01ER45890) is gratefully acknowledged. We also thank James C. Foley, R. Bruce Thompson, and David B. Rehbein at Ames Laboratory for their discussions.

\section{APPENDIX}

For polycrystals of cubic symmetry, the nonzero terms of the eighth-rank covariance, $\Xi_{i j k l}^{\alpha \beta \gamma \delta}$ in Eq. (14), are determined. The first term within the brackets is given as

$$
\begin{aligned}
& \left\langle\sum_{n=1}^{3} a_{i n} a_{j n} a_{k n} a_{l n} \sum_{n=1}^{3} a_{\alpha n} a_{\beta n} a_{\gamma n} a_{\delta n}\right\rangle \\
& =\frac{1}{4 \pi^{2}} \int_{0}^{2 \pi} \int_{0}^{2 \pi} \int_{-1}^{+1}\left(\sum_{n=1}^{3} a_{i n} a_{j n} a_{k n} a_{l n}\right)\left(\sum_{n=1}^{3} a_{\alpha n} a_{\beta n} a_{\gamma n} a_{\delta n}\right) w(\xi, \psi, \varphi) d \xi d \psi d \varphi \\
& =4 \pi^{2}\left\{W_{000} T_{000}+W_{400}\left[T_{400}+\frac{5}{\sqrt{70}}\left(T_{404}+T_{40 \overline{4}}\right)\right]+W_{420}\left[T_{420}+T_{4 \overline{2} 0}+\frac{5}{\sqrt{70}}\left(T_{424}+T_{4 \overline{2} 4}+T_{42 \overline{4}}+T_{4 \overline{2} \overline{4}}\right)\right]\right. \\
& +W_{440}\left[T_{440}+T_{4 \overline{4} 0}+\frac{5}{\sqrt{70}}\left(T_{444}+T_{4 \overline{4} 4}+T_{44 \overline{4}}+T_{4 \overline{4} \overline{4}}\right)\right]+W_{600}\left[T_{600}-\frac{\sqrt{14}}{2}\left(T_{604}+T_{60 \overline{4}}\right)\right] \\
& +W_{620}\left[T_{620}+T_{6 \overline{2} 0}-\frac{\sqrt{14}}{2}\left(T_{624}+T_{6 \overline{2} 4}+T_{62 \overline{4}}+T_{6 \overline{2} \overline{4}}\right)\right]+W_{640}\left[T_{640}+T_{6 \overline{4} 0}-\frac{\sqrt{14}}{2}\left(T_{644}+T_{6 \overline{4} 4}+T_{64 \overline{4}}+T_{6 \overline{4} \overline{4}}\right)\right] \\
& +W_{660}\left[T_{660}+T_{6 \overline{6} 0}-\frac{\sqrt{14}}{2}\left(T_{664}+T_{6 \overline{6} 4}+T_{66 \overline{4}}+T_{6 \overline{6} \overline{4}}\right)\right]+W_{800}\left[T_{800}+\frac{\sqrt{154}}{33}\left(T_{804}+T_{80 \overline{4}}\right)+\frac{\sqrt{1430}}{66}\left(T_{808}+T_{80 \overline{8}}\right)\right]
\end{aligned}
$$




$$
\begin{aligned}
& +W_{820}\left[T_{820}+T_{8 \overline{2} 0}+\frac{\sqrt{154}}{33}\left(T_{824}+T_{8 \overline{2} 4}+T_{82 \overline{4}}+T_{8 \overline{2} \overline{4}}\right)+\frac{\sqrt{1430}}{66}\left(T_{828}+T_{8 \overline{2} \overline{8}}+T_{82 \overline{8}}+T_{8 \overline{2} \overline{8})}\right]\right. \\
& +W_{840}\left[T_{840}+T_{8 \overline{4} 0}+\frac{\sqrt{154}}{33}\left(T_{844}+T_{8 \overline{4} 4}+T_{84 \overline{4}}+T_{8 \overline{4} \overline{4}}\right)+\frac{\sqrt{1430}}{66}\left(T_{848}+T_{8 \overline{4} \overline{8}}+T_{84 \overline{8}}+T_{8 \overline{4} \overline{8})}\right]\right. \\
& +W_{860}\left[T_{860}+T_{8 \overline{6} 0}+\frac{\sqrt{154}}{33}\left(T_{864}+T_{8 \overline{6} 4}+T_{86 \overline{4}}+T_{8 \overline{6} \overline{4})}+\frac{\sqrt{1430}}{66}\left(T_{868}+T_{8 \overline{6} 8}+T_{86 \overline{8}}+T_{8 \overline{6} \overline{8})}\right]\right.\right. \\
& +W_{880}\left[T_{880}+T_{8 \overline{8} 0}+\frac{\sqrt{154}}{33}\left(T_{884}+T_{8 \overline{8} 4}+T_{88 \overline{4}}+T_{8 \overline{8} \overline{4})}\right)+\frac{\sqrt{1430}}{66}\left(T_{888}+T_{8 \overline{8} 8}+T_{88 \overline{8}}+T_{8 \overline{8} \overline{8})}\right]\right\},
\end{aligned}
$$

where the $w$ is orientation distribution function (ODF). It can be expanded in a series of generalized spherical harmonics, with the coefficients $W_{l m n}$ defining the orientation distribution coefficients (ODCs). The notations $T_{l m n}$ are defined as

$$
T_{l m n}=\frac{1}{4 \pi^{2}} \int_{0}^{2 \pi} \int_{0}^{2 \pi} \int_{-1}^{+1}\left(\sum_{n=1}^{3} a_{i n} a_{j n} a_{k n} a_{l n}\right)\left(\sum_{n=1}^{3} a_{\alpha n} a_{\beta n} a_{\gamma n} a_{\delta n}\right) \times Z_{l m n}(\xi) e^{-i m \psi} e^{-i n \varphi} d \xi d \psi d \varphi .
$$

If the polycrystal is of orthorhombic-cubic symmetry, an example term $(i=j=k=l=1$ and $\alpha=\beta=\gamma=\delta=1)$ is given as follows:

$$
\begin{aligned}
& \left\langle\sum_{n=1}^{3} a_{n 1}^{4} \sum_{m=1}^{3} a_{m 1}^{4}\right\rangle=\frac{41}{105}+\frac{2232 \sqrt{2} \pi^{2}}{5005} W_{400}-\frac{2976 \sqrt{5} \pi^{2}}{5005} W_{420}-\frac{1448 \sqrt{35} \pi^{2}}{5005} W_{440}-\frac{12 \sqrt{26} \pi^{2}}{1001} W_{600}+\frac{64 \sqrt{2730} \pi^{2}}{15015} W_{620} \\
& \quad-\frac{128 \sqrt{91} \pi^{2}}{5005} W_{640}+\frac{64 \sqrt{6006} \pi^{2}}{15015} W_{660}+\frac{56 \sqrt{34} \pi^{2}}{7293} W_{800}-\frac{32 \sqrt{1190} \pi^{2}}{12155} W_{820}+\frac{32 \sqrt{1309} \pi^{2}}{12155} W_{840}-\frac{32 \sqrt{14586} \pi^{2}}{36465} W_{860} \\
& \quad+\frac{16 \sqrt{12155} \pi^{2}}{12155} W_{880} .
\end{aligned}
$$

The remaining nonzero terms necessary for the calculation are given in detail elsewhere (Yang, 2003).

Ahmed, S., and Thompson, R. B. (1992). "Effects of preferred grain orientation and grain elongation on ultrasonic wave propagation in stainless steel," in Review of Progress in Quantitative NDE, edited by D. O. Thompson and D. E. Chimenti (Plenum, New York), Vol. 11, pp. 1999-2006. Ahmed, S., and Thompson, R. B. (1996). "Propagation of elastic waves in equiaxed stainless-steel polycrystals with aligned [001] axes," J. Acoust. Soc. Am. 99, 2086-2096.

Bunge, H. J. (1982). Texture Analysis in Materials Science: Mathematical Methods (Butterworths, London).

Frisch, U. (1968). "Wave propagation in random media," in Probabilistic Methods in Applied Mathematics, edited by A. T. Barucha-Reid (Academic, New York), Vol. 1, pp. 75-198.

Hirao, M., Aoki, K., and Fukuoka, H. (1987). "Texture of polycrystalline metals characterized by ultrasonic velocity measurements," J. Acoust. Soc. Am. 81, 1434-1440.

Hirsekorn, S. (1982). "The scattering of ultrasonic waves by polycrystals," J. Acoust. Soc. Am. 72, 1021-1031.

Hirsekorn, S. (1983). "The scattering of ultrasonic waves by polycrystals. II. Shear waves," J. Acoust. Soc. Am. 73, 1160-1163.

Hirsekorn, S. (1985). "The scattering of ultrasonic waves in polycrystalline materials with texture," J. Acoust. Soc. Am. 77, 832-843.

Hirsekorn, S. (1986). "Directional dependence of ultrasonic propagation in textured polycrystals,” J. Acoust. Soc. Am. 79, 1269-1279.

Johnson, G. C. (1985). “Acoustoelastic response of a polycrystalline aggre- gates with orthotropic texture," J. Appl. Mech. 52, 659-663.

Karal, F. C., and Keller, J. B. (1964). "Elastic, electromagnetic, and other waves in a random medium," J. Math. Phys. 5, 537-547.

Li, Y., and Thompson, R. B. (1990). "Relations between elastic constants $C_{i j}$ and texture parameters for hexagonal materials," J. Appl. Phys. 67, 2663-2665.

Roe, R.-J. (1965). "Description of crystallite orientation in polycrystalline materials. III. General solution to pole figure inversion," J. Appl. Phys. 36, 2024-2031.

Roe, R.-J. (1966). "Inversion of pole figures for materials having cubic crystal symmetry,” J. Appl. Phys. 37, 2069-2072.

Sayers, C. M. (1982). "Ultrasonic velocities in anisotropic polycrystalline aggregates," J. Phys. D: Appl. Phys. 15, 2157-2167.

Stanke, F. E., and Kino, G. S. (1984). "A unified theory for elastic wave propagation in polycrystalline materials," J. Acoust. Soc. Am. 75, 665681.

Turner, J. A. (1999). "Elastic wave propagation and scattering in heterogeneous, anisotropic media: Textured polycrystalline materials," J. Acoust. Soc. Am. 106, 541-552.

Weaver, R. L. (1990). "Diffusion of ultrasound in polycrystals," J. Mech. Phys. Solids 38, 55-86.

Yang, L. (2003). "Scattering of elastic waves in statistically anisotropic media," Ph.D. thesis, University of Nebraska. 